\title{
(6) OPEN ACCESS \\ Do variations in hospital mortality patterns after weekend admission reflect reduced quality of care or different patient cohorts? \\ A population-based study
}

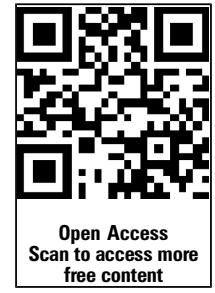

- Additional material is published online only. To view please visit the journal online (http://dx.doi.org/10.1136/bmjqs2013-002218)

${ }^{1}$ Centre for Health Informatics, Australian Institute of Health Innovation, University of New South Wales, Kensington, New South Wales, Australia

${ }^{2}$ SWS Clinical School, Liverpool Hospital, University of New South Wales, Kensington, New South Wales, Australia ${ }^{3}$ The Simpson Centre for Health Services Research, Australian Institute of Health Innovation, University of New South Wales, Kensington, New South Wales, Australia

${ }^{4}$ Sydney South West Local Health District, Department of Radiation Oncology, Cancer Therapy Centre, Liverpool Hospital, Liverpool, New South Wales, Australia

\section{Correspondence to} Professor Enrico Coiera, Centre for Health Informatics, Australian Institute of Health Innovation, Level 1, AGSM Building, G27, University of New South Wales, Kensington, NSW 2052, Australia; e.coiera@unsw. edu.au

Received 9 June 2013 Revised 15 September 2013 Accepted 21 September 2013 Published Online First 25 October 2013

To cite: Concha $\mathrm{OP}$ Gallego B, Hillman K, et al. BMJ Qual Saf 2014;23:

215-222. 
Table 1 Summary of some relevant studies providing evidence for the weekend effect

\begin{tabular}{|c|c|c|c|}
\hline Author & Odds ratio & $95 \% \mathrm{Cl}$ & Group of patients under study (adjustments) \\
\hline Aylin et al ${ }^{1}$ & 1.10 & 1.08 to 1.11 & All patients to emergency department (age, sex, socioeconomic deprivation, comorbidity and diagnosis). \\
\hline Bell et $\left.a\right|^{3}$ & 1.28 & 1.13 to 1.46 & Ruptured abdominal aortic aneurysm (age, sex and Charlson comorbidity index) \\
\hline Bell et $a \beta^{3}$ & 5.28 & 1.01 to 27.50 & Acute epiglotitis (age, sex and Charlson comorbidity index) \\
\hline Bell et $a \beta^{3}$ & 1.19 & 1.03 to 1.36 & Pulmonary embolism (age, sex and Charlson comorbidity index) \\
\hline Palmer et al ${ }^{11}$ & 1.26 & 1.16 to 1.37 & $\begin{array}{l}\text { Stroke patients and 7-day in-hospital mortality for Sunday admissions with Monday as a reference (age, } \\
\text { sex, socioeconomic deprivation quintile, number of previous admissions, comorbidities, month of } \\
\text { discharge, ethnic group, source of admission and stroke type) }\end{array}$ \\
\hline James et $a l^{12}$ & 1.07 & 1.02 to 1.12 & $\begin{array}{l}\text { Acute kidney injury (age, sex, race, Charlson comorbidity index and requirement for mechanical } \\
\text { ventilation) }\end{array}$ \\
\hline \multirow{2}{*}{$\begin{array}{l}\text { Aujesky } \\
\text { et } a l^{13}\end{array}$} & 1.17 & 1.03 to 1.34 & Pulmonary embolism and 30 days post-discharge (age, sex, and the Charlson comorbidity index) \\
\hline & Hazard ratio & & \\
\hline Fang et $a l^{9}$ & 1.12 & 1.00 to 1.25 & Stroke patients and 7 days post-discharge (age, sex, stroke severity and comorbidity) \\
\hline $\begin{array}{l}\text { McKinney } \\
\text { et } a l^{10}\end{array}$ & 1.05 & 1.02 to 1.09 & $\begin{array}{l}\text { Stroke patients and } 90 \text { days post-discharge (patient demographics, coexisting conditions and treatment } \\
\text { with intravenous thrombolysis) }\end{array}$ \\
\hline
\end{tabular}

heterogeneity in prior studies may reflect the window over which deaths are counted. While most weekend effect studies do not count deaths post-discharge, three studies that did found a significant increase in the risk of death at 7 days, ${ }^{9} 30$ days $^{14}$ and 90 days ${ }^{10}$ post-discharge (table 1).

One approach to untangling the relative contribution of patient condition and quality of care is to study the temporal unfolding of the weekend effect on the risk of death per diagnostic group. There are essentially four basic temporal patterns that can be hypothesised from first principles (figure 1). If there is no difference between the cohorts admitted on weekdays and weekends and care is identical for both, then we should see no differences in deaths between the two ( $\mathrm{H} 0$, figure 1$)$. If the weekend cohort has an intrinsically higher probability of dying independent of the care they receive (patient effect), then its pattern of excess mortality should be steady over the short run and not reduce when re-exposed to weekday care $(\mathrm{H} 2$, figure 1$)$. If the quality of weekend care is the cause of excess mortality (care effect), then a bump in death risk should diminish after re-exposure to weekday care $(\mathrm{H} 1$, figure 1). The care effect bump might vary in onset, size and duration. If weekend patients are both sicker and receive poorer care, we would expect a mixed picture with evidence of a temporally localised increase in mortality, settling to a level reflecting the differences in patient condition between both cohorts (H3, figure 1).

In this study the impact of the weekend effect on mortality is examined to see if there are such variations in the 7 days post-admission patterns of risk between weekday and weekend admission patients and, if so, whether they can shed light on the complex association of excess mortality and the weekend admission to hospital.
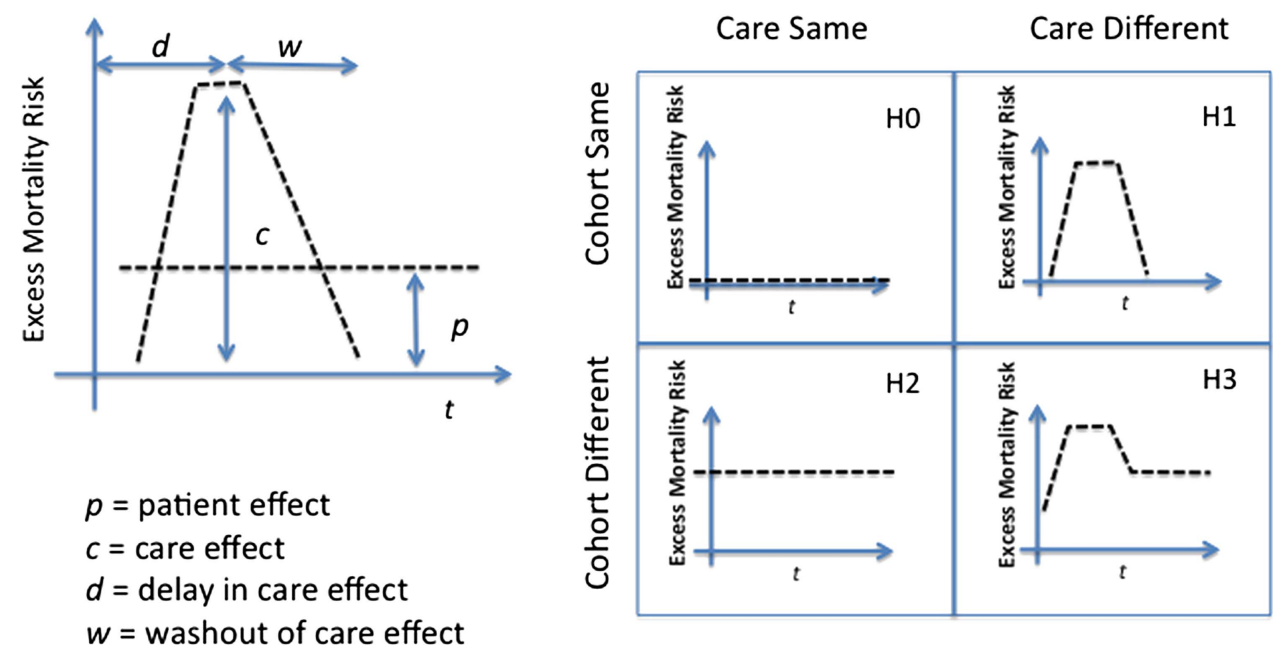

Figure 1 Excess mortality risk associated with weekend admission per diagnosis related group may exhibit different temporal patterns depending on whether the cause is sicker patients (patient effect: H2) or poorer care (care effect: H1). 


\section{METHODS}

All 3798599 admissions through the ED to all 501 public and private hospitals in New South Wales (NSW), Australia between 1 July 2000 and 30 June 2007 were extracted from the NSW Admitted Patient Data Collection. To capture post-discharge deaths, records were linked to the death registry by the NSW Centre for Health Record Linkage. ${ }^{20}$ Clinical information was coded using the International Classification of Diseases 10th revision Australian modification (ICD-10-AM) and Australian refined diagnosis related group (DRG) codes. ${ }^{21}$

Admission in this study refers to an episode of care that ends in discharge, transfer, death or change in care type. Records with an invalid or missing date of admission, date of death, principal diagnosis, DRG, patient age or sex were excluded. If a patient was hospitalised and readmitted within 1 week of discharge, only the last episode of care is taken into account. The resulting data contain 3381962 admissions and 64789 deaths within 1 week from admission across 539122 patients (average of 6.3 admissions per patient over 7 years). The Charlson comorbidity index was calculated for each admission using ICD-10-AM codes. 223

\section{Statistical analysis}

To minimise selection bias, admissions were stratified by age, sex, Charlson comorbidity index and DRG. Thus variations in illness severity within a diagnostic and comorbidity group remained unadjusted, since they cannot be estimated from hospital administrative datasets.

A weekend was defined as the period between midnight Friday and midnight Sunday. ${ }^{3}{ }^{11}$ Mortality rates in the week following admission were estimated by direct standardisation for both weekend and weekday admissions. ${ }^{12}{ }^{24}$ Gamma methods were used for the CIs of the mortality rates. ${ }^{25}$ For each DRG, odd ratios (ORs) of death following weekend versus weekday admissions were computed using Mantel-Haenszel statistics. ${ }^{26}$ Survival curves and hazard ratios (HRs) were calculated for each DRG using Cox statistics. ${ }^{27}$ Excess mortality risk curves, $e(t)$, were the result of subtracting the probabilities of dying, $f(t)$, if admitted on weekend or weekday, $e(t)=\left(f_{\text {weekend }}(t)-f_{\text {weekday }}(t)\right)$, at each day post-admission from admission to day 7 after admission, $\mathrm{t}=0 . . .7$.

Excess deaths for each DRG were calculated as the product of cumulative excess mortality risk and the corresponding number of weekend admissions for that DRG. This represents the number of deaths that could be avoided if there was no weekend effect.

A DRG was categorised as exhibiting a weekend effect if (1) its OR>1, HR>1 and 95\% CI intervals were $>1$ and $p$ values $<0.01$; (2) overall number of deaths $>50$; (3) cumulative excess mortality risk in the week post-admission $\geq 0.005$ (5 extra deaths per
1000 weekend admissions). To test for difference in the shape of excess mortality risk curves, root mean square errors between curves were calculated.

In addition, Mantel-Haenszel statistics were calculated separately for in-hospital and post-discharge deaths. The latter only considered patients discharged within 1 week after admission. Statistical analyses were performed with the use of SAS V.9.0 and IBM-SPSS V.20 software.

\section{RESULTS}

The mean age of patients was 49 years (median $=52.4$, percentile $25=26.8$, percentile $75=74.6, \mathrm{SD}=45.6$ ) and the mean length of stay was 3.8 days (median $=1.0$, percentile $25=0.0$, percentile $75=5.0$, $\mathrm{SD}=8.3$ ). Weekends accounted for $27.1 \%$ of all admissions (917 257/3 381 962) and these admissions were associated with $28.2 \%$ of all deaths (18 282/ 64 789). There were statistically significant differences in the characteristics of patients admitted on weekdays and weekends (table 2). After adjusting for these differences, the mortality rate for weekday admissions fell from $1.92 \%$ (46 507 deaths) to $1.85 \%$ (95\% CI $1.85 \%$ to $1.85 \%$ ) and for weekend admissions rose from $2.03 \%$ to $2.12 \%$, (95\% CI $2.12 \%$ to $2.12 \%$ ) (difference $0.27 \%, \mathrm{p}<0.001$ ). The main drivers for adjustment were the greater age and comorbidity scores of patients admitted on weekdays.

After adjusting by age, sex and comorbidity index, no DRG (of a total of 430) was associated with a reduced risk of death following weekend admissions. Sixteen of the 430 DRGs demonstrated a weekend effect (table 3 and supplementary table S1 in online

Table 2 Baseline characteristics of patients admitted on weekdays and weekend

\begin{tabular}{|c|c|c|c|}
\hline & $\begin{array}{l}\text { Weekday } \\
\mathrm{N}=2464705 \\
(72.9 \%) \%\end{array}$ & $\begin{array}{l}\text { Weekend } \\
N=917257 \\
(27.1 \%) \%\end{array}$ & $\chi^{2}$ ( $\mathrm{p}$ value $)$ \\
\hline \multicolumn{4}{|l|}{ Age (years) } \\
\hline $0-5$ & 8.2 & 8.9 & \multirow{8}{*}{$\begin{array}{l}6575.7 \\
(<0.001)\end{array}$} \\
\hline $6-15$ & 5.6 & 6.5 & \\
\hline $16-35$ & 18.3 & 21.3 & \\
\hline $36-55$ & 19.3 & 19.0 & \\
\hline $56-65$ & 10.6 & 9.9 & \\
\hline $66-75$ & 12.9 & 11.8 & \\
\hline $76-85$ & 16.3 & 14.8 & \\
\hline$\geq 85$ & 8.6 & 7.9 & \\
\hline \multicolumn{4}{|c|}{ Charlson comorbidity score } \\
\hline Zero to mild $(0)$ & 51.7 & 55.6 & \multirow{4}{*}{$\begin{array}{l}4477.7 \\
(<0.001)\end{array}$} \\
\hline Mild (1-2) & 26.1 & 24.7 & \\
\hline Moderate (3-4) & 11.2 & 10.0 & \\
\hline Severe $(\geq 5)$ & 11.0 & 9.7 & \\
\hline \multicolumn{4}{|l|}{ Sex } \\
\hline Women & 50.3 & 51.9 & \multirow{2}{*}{$\begin{array}{l}645.8 \\
(<0.001)\end{array}$} \\
\hline Men & 49.7 & 48.1 & \\
\hline
\end{tabular}


appendix). These 16 DRGs accounted for $39.7 \%$ (25 695/64 789) of all deaths, 40.1\% (7325/18 282) of weekend deaths (21 excess deaths per 1000 admissions). Taken as a combined group, these 16 DRGs exhibited a mixed pattern $(\mathrm{H} 3$, figure 1 with delay $\mathrm{d}=0$ ) consistent with both patient effect and weekend care contributing to excess mortality (supplementary figure $\mathrm{S} 1$ in online appendix).

Analysis of individual excess mortality risk curves for these 16 DRGs revealed a variety of temporal patterns (figure 2 and supplementary figure S2 in online appendix):

1. Care effect (H1): Two patterns demonstrated a brief rise in mortality associated with weekend admission:

- An early risk pattern in which the majority of excess deaths occurred within the first $24 \mathrm{~h}$ after admission. The only DRG in this category was F70 (Major Arrhythmia and Cardiac Arrest).

- A risk washout pattern showed the greatest impact of weekend admission in the first $48 \mathrm{~h}$, gradually reducing throughout the rest of the week. The three diagnoses in this category were E61 (Pulmonary Embolism), E64 (Pulmonary Oedema and Respiratory Failure) and F65 (Peripheral Vascular Disorders).

2. Patient effect (H2): With the steady risk pattern, the impact of weekend admission was distributed uniformly across the week after admission and appeared not to alter with re-exposure to weekday care. DRGs with this pattern were I65 (Connective Tissue Malignancy, including Pathological Fracture), R60 (Acute Leukemia) and R61 (Lymphoma and Non-Acute Leukaemia).

\section{Care Effect: Early Risk}
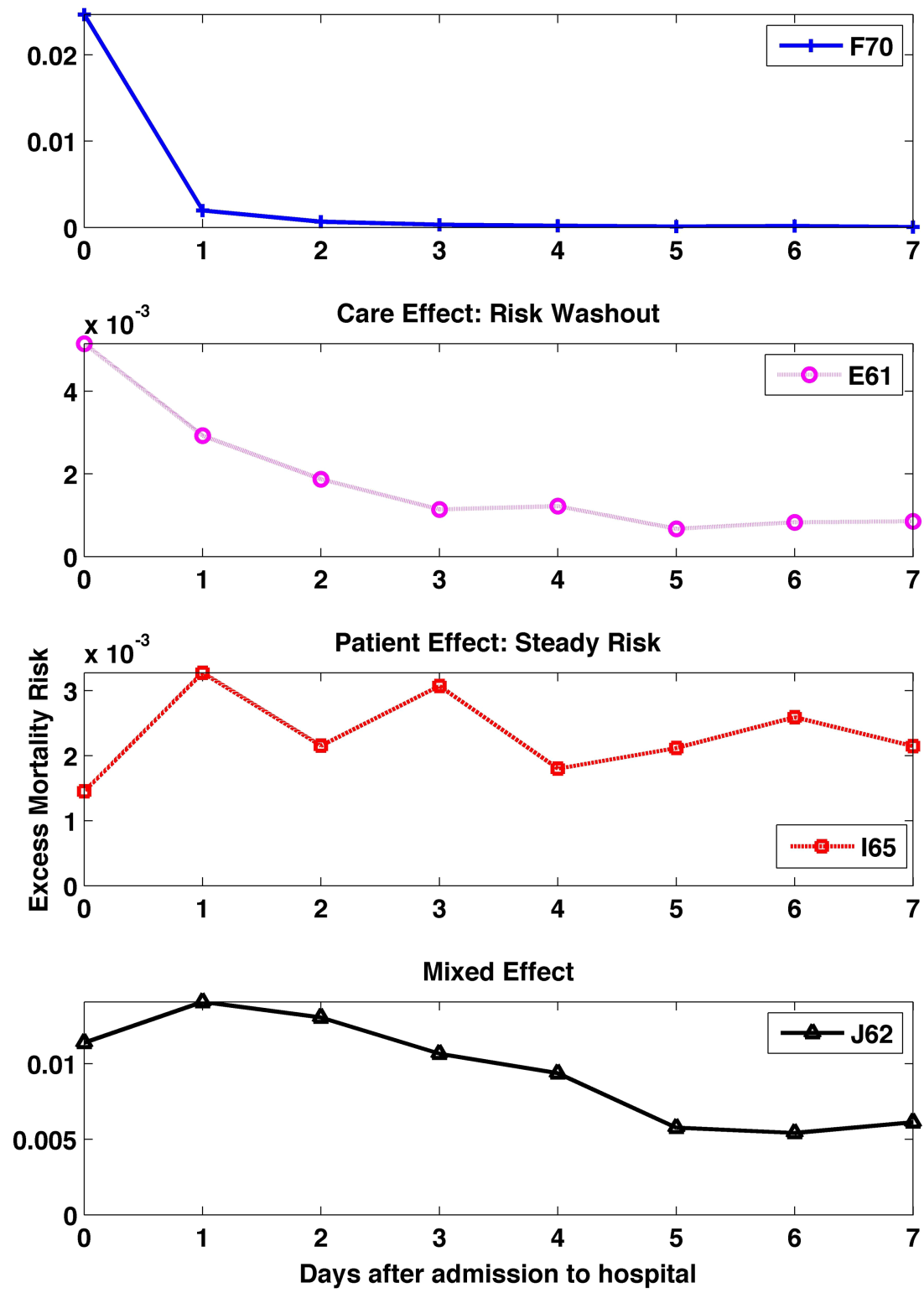

Figure 2 Excess mortality risks curves for selected diagnosis related groups (DRGs). Excess mortality risk curves for selected DRGs consistent with care effect (top two panels), patient effect (panel 3) and mixed effect (bottom panel). 
3. Mixed effect (H3): In this group a spike in mortality was associated with weekend admission and reduced on exposure to weekday care, but remained elevated after washout. DRGs in this group were B02 (Craniotomy), B67 (Degenerative Nervous System Disorders), B70 (Stroke and Other Cerebrovascular Disorders), E71 (Respiratory Neoplasms), F62 (Heart Failure and Shock), G60 (Malignancy), H61 (Malignancy of Hepatobiliary System, Pancreas), J62 (Malignant Breast Disorders) and L60 (Renal Failure).

For some DRGs there were significantly higher numbers of post-discharge deaths following weekend admissions. For example, B02 (Craniotomy) had a postdischarge mortality rate of $5.0 \%$ for weekend admissions versus $2.7 \%$ for weekday admissions. However, after adjustment, no DRG had post-discharge only ORs with $\mathrm{p}$ values $<0.01$ (table 3 ).

\section{DISCUSSION}

In this study, different diagnostic groups showed varying temporal patterns of excess mortality following weekend admission. Variations in patient illness, severity and comorbidity between weekday and weekend admissions are widely acknowledged confounders in studies of the weekend effect, often criticised for ascribing to clinical care what might be due to biology. As in other studies, ${ }^{9} 1014$ the impact of weekend admission clearly extends beyond the hospital walls and lingers over time. Indeed, our data show that the effect of weekend admission on mortality has not saturated at 1 week after admission (figure 2 and supplementary figure S1 in online appendix), so it is very likely that additional deaths occur well beyond that window.

Only 16 of 430 DRGs had a significant increased risk of death following weekend admission. Within each DRG, the probability of the weekend effect being a false positive (independently of any other DRG) is less than $1 \%$. The probability that the weekend effect is a false positive in at least one of the 16 DRGs is $p=1-(1-p)^{16}=15 \%$.

These 16 DRGs displayed all of the variations in the 7-day post-admission temporal pattern hypothesised from first principles. The early risk pattern, where most of the risk of death is within the first $24 \mathrm{~h}$ of admission, was associated with cardiac arrest and arrhythmia. Others have also demonstrated weekend admission effects for patients with myocardial infarction. ${ }^{15}$ Failure to provide immediate care to manage these conditions can have an immediate and fatal outcome, probably explaining the pattern. Although some disagree, ${ }^{2}$ patients in this category are as likely to be admitted to hospital on any day of the week, so the weekend effect most probably is due to variation in care-for example, unavailability of specialist staff, imaging or stenting services. While our population includes some centres of excellence in cardiac care that offer services across the weekend, it also includes smaller and rural hospitals where access is diminished on weekends.

In the risk washout pattern, the effect of weekend admission clearly dominates the first $48 \mathrm{~h}$, but gradually 'washes out' of the system over time, and was associated with DRGs for pulmonary embolism, pulmonary oedema and peripheral vascular disorders. These are also acute events requiring access to high quality immediate care, but with a less abrupt risk of immediate mortality. One reading of this pattern is that those who survive the first $48 \mathrm{~h}$ fare better when re-exposed to weekday care.

Cancer patients dominated the steady risk pattern, with a similar mortality risk across the week after admission. In common with other weekend effect studies that rely on population level administrative data, we were unable to distinguish illness severity within a DRG and comorbidity group. It is therefore possible that cancer patients with more severe illness were indeed admitted on the weekend-for example, when community care could no longer manage them. If so, this may explain the pattern seen here, where the risk of death did not change when patients admitted at the weekend were re-exposed to weekday care.

Finally, a group of DRGs exhibited a mixed effect pattern expected when both quality of care and patient effect contribute to excess mortality. This pattern was associated with a mixture of diagnoses such as stroke, nervous system disorders and some cancers. This was also the pattern shown by the group of 16 DRGs taken as a whole.

If a patient was readmitted within one week of discharge, only the last episode of care was taken into account in our analysis. The rationale for this was that the last admission is the one where death might occur or is closest in time to death. To test for distortions introduced by this choice, we repeated our analysis but based it on DRGs associated with the first admission in a sequence. The results of this analysis were nearly identical to the original analysis, with only B67 (Degenerative Nervous System Disorders) no longer exhibiting a weekend effect $(p=0.02)$. The four patterns of excess mortality risk curves remained the same, with no new patterns or new DRGs emerging with a weekend effect. Admission to the ICU during the hospital stay can be used to help identify patients who are critically ill. In this study, treating ICU admission as a confounder gave similar results for all patterns with B67 again dropping out of the weekend effect group $(\mathrm{p}=0.03)$.

While we defined a weekend as the period between midnight Friday and midnight Sunday, any time outside 08:00 h and 18:00 h Monday to Friday might be considered not part of 'normal' operating hours and also contribute to deviations in observed mortality. Furthermore, the observed increase in mortality was not homogeneous across these non-core operating times. In particular, adjusted mortality for our 16 selected DRGs 
Table 3 DRGs at risk of weekend effect: number of weekday/weekend admissions and deaths, Odds Ratios (OR), Hazard Ratios (HR). OR are broke down into in-hospital and post-discharge ratios

\begin{tabular}{|c|c|c|c|c|c|c|c|c|c|c|c|c|c|}
\hline \multirow[b]{2}{*}{ DRG } & \multicolumn{2}{|c|}{ Admissions } & \multicolumn{3}{|l|}{ Deaths } & \multicolumn{4}{|c|}{ In-hospital and Post-discharge } & \multicolumn{2}{|c|}{ In-hospital only } & \multicolumn{2}{|c|}{ Post-discharge only } \\
\hline & Weekday & Weekend & Weekday & Weekend & $\begin{array}{l}\text { Excess Deaths } \\
(95 \% \mathrm{Cl})\end{array}$ & $\mathrm{p}$ Value & OR $(95 \% \mathrm{Cl})$ & $\mathrm{p}$ Value & HR $(95 \% \mathrm{Cl})$ & $\mathrm{p}$ Value & OR $(95 \% \mathrm{Cl})$ & $\mathrm{p}$ Value & OR $(95 \% \mathrm{Cl})$ \\
\hline B02, Craniotomy & 3475 & 965 & 256 & 101 & $26(18-36)$ & .001 & $1.51(1.18-1.94)$ & .002 & $1.44(1.14-1.81)$ & .003 & $1.47(1.14-1.89)$ & .042 & $4.48(1.06-18.91)$ \\
\hline $\begin{array}{l}\text { B67, Degenerative nervous system } \\
\text { disorders }\end{array}$ & 6192 & 1836 & 267 & 105 & $26(18-36)$ & .005 & $1.40(1.1-1.77)$ & .004 & $1.39(1.11-1.74)$ & .004 & $1.44(1.12-1.83)$ & .987 & $1.11(0.47-2.63)$ \\
\hline $\begin{array}{l}\text { B70, Stroke and other } \\
\text { cerebrovascular disorders }\end{array}$ & 36762 & 13274 & 4627 & 1855 & $165(137-195)$ & $<.001$ & $1.13(1.07-1.2)$ & $<.001$ & $1.12(1.06-1.18)$ & $<.001$ & $1.13(1.07-1.2)$ & .166 & $1.15(0.95-1.38)$ \\
\hline E61, Pulmonary embolism & 12123 & 2963 & 409 & 153 & $44(33-57)$ & $<.001$ & $1.59(1.31-1.93)$ & $<.001$ & $1.53(1.27-1.84)$ & $<.001$ & $1.64(1.34-1.99)$ & .991 & $1.13(0.45-2.82)$ \\
\hline $\begin{array}{l}\text { E64, Pulmonary oedema and } \\
\text { respiratory failure }\end{array}$ & 4635 & 1734 & 941 & 413 & $61(48-75)$ & .001 & $1.25(1.09-1.43)$ & .001 & $1.21(1.08-1.36)$ & .002 & $1.24(1.08-1.42)$ & .405 & $1.28(0.77-2.13)$ \\
\hline E71, Respiratory neoplasms & 9648 & 2895 & 1658 & 672 & $172(151-195)$ & $<.001$ & $1.45(1.31-1.61)$ & $<.001$ & $1.39(1.27-1.52)$ & $<.001$ & $1.43(1.29-1.59)$ & .013 & $1.50(1.1-2.04)$ \\
\hline F62, Heart failure and shock & 45191 & 14598 & 2476 & 932 & $120(96-146)$ & $<.001$ & $1.17(1.08-1.27)$ & $<.001$ & $1.17(1.08-1.26)$ & $<.001$ & $1.16(1.07-1.26)$ & .066 & $1.32(0.99-1.75)$ \\
\hline F65, Peripheral vascular disorders & 7457 & 2069 & 844 & 310 & $38(18-74)$ & $<.001$ & $1.37(1.18-1.59)$ & $<.001$ & $1.31(1.15-1.5)$ & $<.001$ & $1.38(1.18-1.62)$ & .196 & $1.31(0.9-1.91)$ \\
\hline $\begin{array}{l}\text { F70, Major arrhythmia and cardiac } \\
\text { arrest }\end{array}$ & 7268 & 2842 & 3157 & 1326 & $98(82-113)$ & .004 & $1.14(1.04-1.25)$ & .009 & $1.10(1.02-1.16)$ & .004 & $1.14(1.04-1.25)$ & .727 & $1.07(0.79-1.44)$ \\
\hline G60, Digestive malignancy & 5244 & 1571 & 904 & 365 & $92(74-114)$ & $<.001$ & $1.45(1.26-1.67)$ & $<.001$ & $1.40(1.24-1.59)$ & $<.001$ & $1.48(1.29-1.71)$ & .326 & $1.25(0.83-1.89)$ \\
\hline $\begin{array}{l}\text { H61, Malignancy of hepatobiliary } \\
\text { system, pancreas }\end{array}$ & 4209 & 1253 & 822 & 338 & $90(60-127)$ & $<.001$ & $1.54(1.33-1.78)$ & $<.001$ & $1.44(1.27-1.64)$ & $<.001$ & $1.49(1.28-1.74)$ & .025 & $1.68(1.09-2.6)$ \\
\hline $\begin{array}{l}\text { 165, Connective tissue malignancy, } \\
\text { including pathological fracture }\end{array}$ & 4415 & 1208 & 246 & 94 & $22(2-151)$ & .005 & $1.43(1.11-1.84)$ & .004 & $1.41(1.11-1.8)$ & .003 & $1.48(1.14-1.93)$ & .667 & $1.27(0.61-2.68)$ \\
\hline J62, Malignant breast disorders & 1382 & 418 & 220 & 97 & $31(23-41)$ & .001 & $1.60(1.22-2.1)$ & $<.001$ & $1.56(1.22-1.98)$ & $<.001$ & $1.73(1.31-2.29)$ & .955 & $0.88(0.36-2.16)$ \\
\hline L60, Renal failure & 8344 & 2313 & 936 & 320 & $44(20-87)$ & .001 & $1.25(1.09-1.44)$ & .002 & $1.23(1.08-1.4)$ & .003 & $1.24(1.07-1.43)$ & .063 & $1.59(1-2.54)$ \\
\hline R60, Acute leukaemia & 2401 & 597 & 176 & 71 & $17(12-25)$ & $<.001$ & $1.82(1.34-2.49)$ & $<.001$ & $1.69(1.28-2.22)$ & $<.001$ & $1.95(1.41-2.68)$ & .92 & $1.17(0.33-4.11)$ \\
\hline $\begin{array}{l}\text { R61, Lymphoma and non-acute } \\
\text { leukaemia }\end{array}$ & 8487 & 1915 & 431 & 173 & $75(61-90)$ & $<.001$ & $1.88(1.56-2.26)$ & $<.001$ & $1.83(1.54-2.19)$ & $<.001$ & $1.98(1.63-2.41)$ & .971 & $1.07(0.55-2.08)$ \\
\hline
\end{tabular}

Excess deaths for each DRGs were calculated as the product of the cumulative excess mortality risk and the corresponding number of weekend admissions for that DRG. 
was highest for admissions between midnight Friday and midnight Saturday and lowest for admissions between midnight Monday and midnight Tuesday.

\section{Limitations of the study}

The associations between weekend admissions and increased mortality reported here do not establish causality but do fit the hypothesised patterns expected with different causal pathways. There are well-known challenges in the clinical interpretation of administrative data, and this challenge is shared by most other studies of the weekend effect. ${ }^{28}$ For example, in common with most previous literature, severity of illness within a diagnostic and comorbidity group, time and date of procedures and staffing information were not available in this study. Our results are also limited by the quality and methods of DRG coding which emphasise the principal diagnosis for admission and may miss critical comorbidities. For example, a cancer patient admitted for an infection may be coded using an 'infection' instead of a cancer DRG. Therefore, when analysing admissions for cancer patients, we only compared admissions where the primary reason for hospitalisation was cancer.

\section{Future work}

The existence, cause and magnitude of the weekend effect have been discussed in the literature for the last 30 years. Given that different patient groups now appear to have widely varying patterns of risk associated with weekend admission, finer-grained studies using clinical datasets are urgently needed, especially to better model severity and comorbidity and its influence on mortality. However, the very large sample size required for this study would not be currently possible with fine-grained clinical data. As large clinical datasets become available, however, finer analyses should be possible. We should be able, for example, to distinguish between rural and metropolitan hospitals and to test for the impact of reduced specialist services and ICU availability in some rural organisations. Also, differences in patients presenting to EDs and hospitals may partly reflect the services and support available to these patients in the community. Future work should include the effect of these services. Finally, unpacking the relationship between the levels of service provided on weekdays and weekends, controlling for any selection bias in weekend presentations, will help us better understand the risks associated with different staffing and service models.

\section{CONCLUSION}

The weekend effect is not a uniform phenomenon but, rather, a complex cluster of different causal pathways, shaping patients with very different diseases presenting at very different clinical services. Not every diagnostic group demonstrates a weekend effect, and those that do are associated with quite different risk profiles, suggestive of different causal pathways. Recognising how these different patterns shape the impact of weekend admissions should lead to more diagnosis- and service-specific analyses and solutions.

Contributors OPC and BG: contributed to conception and design, analysis and interpretation of the data and results, drafting and writing the article, revising it critically for important intellectual content and final approval of the version to be published. KH and GPD: contributed to interpretation of the data and revising the paper critically for important intellectual and content. EC: contributed to supervision of the paper, developed hypotheses, analysis and interpretation of the data and results, as well as drafting and writing the article, revising it critically for important intellectual content and final approval of the version to be published.

Funding This work was funded by National Health and Medical Research Council (NHMRC) Program grant 568612. Competing interests None.

Ethics approval Ethics approval was obtained from the NSW Population and Health Services Research Ethics Committee and the UNSW Human Research Ethics Committee.

Provenance and peer review Not commissioned; externally peer reviewed.

Open Access This is an Open Access article distributed in accordance with the Creative Commons Attribution Non Commercial (CC BY-NC 3.0) license, which permits others to distribute, remix, adapt, build upon this work noncommercially, and license their derivative works on different terms, provided the original work is properly cited and the use is non-commercial. See: http://creativecommons.org/licenses/by$\mathrm{nc} / 3.0$ /

\section{REFERENCES}

1 Aylin P, Yunus A, Bottle A, et al. Weekend mortality for emergency admissions. A large, multicentre study. Qual Saf Health Care 2010;19:213-17.

2 Becker DJ. Weekend hospitalization and mortality: a critical review. Expert Rev Pharmacoeconomics Outcomes Res 2008;8:23-6.

3 Bell CM, Redelmeier DA. Mortality among patients admitted to hospitals on weekends as compared with weekdays. $N$ Engl J Med 2001;345:663-8.

4 Cram P, Hillis SL, Barnett M, et al. Effects of weekend admission and hospital teaching status on in-hospital mortality. Am J Med 2004;117:151-7.

5 Barnett M, Kaboli P, Sirio C, et al. Day of the week of intensive care admission and patient outcomes: a multisite regional evaluation. Med Care 2002;40:530-9.

6 Gould JB, Qin C, Marks AR, et al. Neonatal mortality in weekend vs weekday births. JAMA 2003;289:2958-62.

7 Schmulewitz L, Proudfoot A, Bell D. The impact of weekends on outcome for emergency patients. Clin Med 2005;5:621-5.

8 Wunsch H, Mapstone J, Brady T, et al. Hospital mortality associated with day and time of admission to intensive care units. Intensive Care Med 2004;30:895-901.

9 Fang J, Saposnik G, Silver FL, et al. Association between weekend hospital presentation and stroke fatality. Neurology 2010;75:1589-96.

10 McKinney JS, Deng Y, Kasner SE, et al. Comprehensive stroke centers overcome the weekend versus weekday gap in stroke treatment and mortality. Stroke 2011;42:2403-9.

11 Palmer WL, Bottle A, Davie C, et al. Dying for the weekend: a retrospective cohort study on the association between day of hospital presentation and the quality and safety of stroke care. Arch Neurol 2012;69:1296-302. 
12 Ahmad OB, Boschi-Pinto C, Lopez AD, et al. Age standardization of rates: a new WHO standard. Geneva: World Health Organization, 2001.

13 James MT, Wald R, Bell CM, et al. Weekend hospital admission, acute kidney injury, and mortality. J Am Soc Nephrol 2010;21:845-51.

14 Aujesky D, Jimenez D, Mor MK, et al. Weekend versus weekday admission and mortality after acute pulmonary embolism. Circulation 2009;119:962-8.

15 Kostis WJ, Demissie K, Marcella SW, et al. Weekend versus weekday admission and mortality from myocardial infarction. N Engl J Med 2007;356:1099-109.

16 Halm EA, Chassin MR. Why do hospital death rates vary? N Engl J Med 2001;345:692-4.

17 Foss NB, Kehlet H. Short-term mortality in hip fracture patients admitted during weekends and holidays. Br J Anaesth 2006;96:450-4.

18 Hamilton P, Eschiti VS, Hernandez K, et al. Differences between weekend and weekday nurse work environments and patient outcomes: a focus group approach to model testing. J Perinat Neonatal Nurs 2007;21:331-41.

19 Skinner CA, Riordan RL, Fraser KL, et al. The challenge of locum working arrangements in New South Wales public hospitals. Med J Aust 2006;185:276-8.
20 Lawrence G, Dinh I, Taylor L. The Centre for Health Record Linkage: a new resource for health services research and evaluation. Health Inform Manage J 2008;37:60.

21 Duckett $S$. The development of Australian refined diagnosis related groups: the Australian inpatient casemix classification. Casemix Q 2000;2:115-20.

22 Charlson ME, Pompei P, Ales KL, et al. A new method of classifying prognostic comorbidity in longitudinal studies: development and validation. J Chronic Dis 1987;40:373-83.

23 Quan H, Sundararajan V, Halfon P, et al. Coding algorithms for defining comorbidities in ICD-9-CM and ICD-10 administrative data. Med Care 2005;43:1130-9.

24 Breslow NE, Day NE. Statistical methods in cancer research. Volume II-The design and analysis of cohort studies. IARC Sci Publ 1987(82):1-406.

25 Fay MP, Feuer EJ. Confidence intervals for directly standardized rates: a method based on the gamma distribution. Stat Med 1997;16:791-801.

26 Woolson RF, Bean JA. Mantel-Haenszel statistics and direct standardization. Stat Med 1982;1:37-9.

27 Cox DR. Regression models and life-tables. J R Stat Soc Series B Stat Methodol 1972;34:187-220.

28 Nathan H, Pawlik TM. Limitations of claims and registry data in surgical oncology research. Ann Surg Oncol 2008;15:415-23. 